Academic City University College - Accra Ghana

Society for Multidisciplinary \& Advanced Research Techniques (SMART) Africa

Tony Blair Institute for Global Change

FAIR Forward - Artificial Intelligence for All - Deutsche Gesellschaft für Internationale Zusammenarbeit (GIZ) GmbH

Accra Bespoke Multidisciplinary Innovations Conference (ABMIC)

\title{
Smart Medication for secure health with Internet of Things
}

Onwodi, G.O. \& Udoette, E.

Department of Computer Science

National Open University of Nigeria

FCT Abuja, Nigeria.

E-mail: gonwodi@noun.edu.ng1

Phone Number:+2347032036365 


\title{
Smart Medication for secure health with Internet of Things
}

\author{
Onwodi, G.O. \& Udoette, E.
}

\begin{abstract}
For over a decade, network and technology has been improved to an equal extent which has led to an easy transmission of data over the entire globe. Besides growth and development of Internet of Things in our daily life such as health care and home automation, secured transmission is significant regarding how confidential and secured the information is, to assure the privacy. The data related to the health are acquired from medical devices and monitoring systems. Some information are acquired from the patient. In this project we will be more particular in gathering the information from the patient. They ultimately end up in Electronic Healthcare Records. These records are sent to labs, doctors, nurses and other parties involved. This enables doctors, caregivers to monitor patient's physiological conditions at any time according to the information obtained. Hence, it is based on this that this research work is carried out to determine the level at which the internet has been able to improve the delivery of healthcare services in the healthcare delivery system in Nigeria.
\end{abstract}

Keywords: Internet of Things, Home Automation, Electronic Healthcare Records, medical devices, monitoring systems.

\section{INTRODUCTION}

Health is one of the predominant challenges for humanity. In Nigeria however, according to World Health Organization, chronic diseases are projected to account for $24 \%$ of all deaths which shows that quite a number of people suffer from chronic illness. The advancement in Information and Communication Technology has enabled modern and innovative health care system to address several health related issues. The worldwide revolution of Information and Communication Technology is sweeping through every aspect of human activities. The concept of Internet of Things (IoT) technology allows doctors to monitor effectively and communicate using user friendly interface between connected things.

The Internet of Things (IoT) creates a lot of options convenience, creating awareness and helping people tackle different issues, thereby enhancing flexibility and facilitating a modernized healthcare system. It facilitates proper storage and retrieval of health records, usually an efficient way to enable remote health monitoring and retrieval of patients' information from the systems in the case of emergency. These health systems can range from storage and retrieval of patients past medical records, monitoring heart rates, blood pressure etc. where the doctors can detect patients health condition. To do all of this, smart medication and monitoring system for secure health using Internet of Things (loT) is a priority.

The health care system becomes a better option to document patients' medical records track and retrieve patients' medical information, thus providing efficient health care services to people. Information sharing in the health care system is usually vulnerable to attacks over the Internet because it is an unsecured network. Ensuring security and privacy of patients medical records are necessity in healthcare systems. 
Hence there is the need for various security features which help to confirm identity of individuals and encryption of data while using the system. This leads to the need for establishment of secured and smart health care system which guarantees efficient delivery and maintenance of patients' health and medical records.

\subsection{Background Of Study}

Traditional Medicine is the system of healthcare delivery in Nigeria. Traditional healing and medical practices include herbalists, divine healers, soothsayers, midwives, spiritualists, bonesetters, mental health therapists and surgeons. Presently, these practices remain a viable part of the healthcare system in the country. Western healthcare was introduced in Nigeria by doctors brought by explorers and traders to cater for their own wellbeing. Infrastructural base takes shape through the foundation of hospitals erected mainly by Christian missionaries. Development of nationwide healthcare service in Nigeria through national development plans started from the First Colonial Development plan in 1945 until the Five year Strategic Plan from 2004-2008. Nigeria is still a developing country with serious issues pertaining to healthcare. Poverty and large scale corruption are big obstacles to improvement in the health and wellbeing of the Nigerian population (Browne, 2017).

The Colonial medical service is seen to grow from the modest beginning of barrack medicine and the care of the isolated expatriate communities into attempts at extensive though thin medical coverage of the vast areas of this populous country. Malaria, leprosy, sleeping sickness, and yellow fever provide successive challenges to the doctors whose training fitted them more for curative medicine in the growing centres of population than for preventive medicine and mass treatment in the bush. The saga of Ibadan is told, with the vision (and incomprehensible oversights) that eventually brought into being a first-class teaching centre, to be followed by Lagos, Ife, Enugu-Nsukka, Zaria and perhaps others. The long-forgotten needs of the predominantly rural population are at last brought into the picture as the new emphasis on preventive medicine, nutrition, and the attack on the widespread endemic diseases demand more attention (Browne, 2017). In the 21st Century, health care systems across the world are focusing policy efforts on improving the quality of healthcare delivered to their population. In contrast, healthcare quality improvement in earlier time periods arose from a series of seemingly unrelated incidents and developments.

\subsection{Statement Of Problem}

Most patients have been undergoing a lot of challenges such as queuing up to see a doctor and most times do not even get to see the doctor as a result of the crowd and as such they go through a lot of stress. However some hospitals move patient's documents or file from one place to the other and before it gets to the doctor. Issues like this, delays the process of giving attention to most patients. It is in this view that a flexible and more efficient system is needed to be developed to ensure improved process. A software application will be designed and developed which will deliver a simpler, improved and more comfortable health care delivery system.

\section{RELATED WORKS}

Healthcare provision in Nigeria is a concurrent responsibility of the three tiers of government in the country. Private providers of healthcare have a visible role to play in health care delivery. The use of Traditional Medicine (TM) and Complementary and Alternative Medicine (CAM) has increased significantly over the past few years. 
In area of health infrastructure the Federal Government 's role is mostly limited to coordinating the affairs of the university teaching hospitals, Federal Medical Centres (tertiary healthcare) while the State government manages the various General Hospitals (Secondary healthcare) and the local government focus on dispensaries (Primary healthcare), which are regulated by the Federal Government (Welcome 2011).

The total expenditure on healthcare as percentage of GDP is $4.6 \%$, while the percentage of Federal Government expenditure on healthcare is about 1.5\%. A long run indicator of the ability of the country to provide food sustenance and avoid malnutrition is the rate of growth of per capita food production; from 1970-1990, the rate for Nigeria was $0.25 \%$. Though small, the positive rate of per capita may be due to Nigeria's importation of food products. Historically, health insurance in Nigeria can be applied to a few instances: free health care provided and financed for all citizens, health care provided by government through a special health insurance scheme for government employees and private firms entering contracts with private health care providers. However, there are few people who fall within the three instances; as at 2015 less than $5 \%$ of Nigerians have health insurance coverage.

In May 1999, the government created the National Health Insurance Scheme, the scheme encompasses government employees, the organized private sector and the informal sector. Legislativewise, the scheme also covers children under five, permanently disabled persons and prison inmates. In 2004, the administration of Obasanjo further gave more legislative powers to the scheme with positive amendments to the original 1999 legislative act. The number of Nigerians covered by the National Health Insurance Scheme (NHIS) since its establishment are 1.5 percent of the population. In 2017, the House of Representatives Committee on Health Care Services in Abuja, organized a two-day investigative hearing; where the Minister of Health Isaac Folorunsho Adewole said that the sum of N351 billion had been expended on Health Management Organizations (HMOs) so far without commensurate result (Welcome, 2011).

There is immense private sector participation in the scheme with HMOs like Health Partners HMO, Total Health Trust, Police HMO, Clearline HMO, Multi Shield Nigeria, Expatcare Health International, Oceanic Health Management and Zuma Health Trust. In 1989 legislation made effective a list of essential drugs. The regulation was also meant to limit the manufacture and import of fake or sub-standard drugs and to curtail false advertising. However, the section on essential drugs was later amended. In 2005 it was estimated that about $16.7 \%$ of pharmaceutical drugs in the country were counterfeit. In 2012 a new study concluded that the proportion had fallen to $6.4 \%$, of which $19.6 \%$ were Anti-Malaria medicines. In 2014 that had fallen to 3.6\%. About N29 billion worth of counterfeit drugs were destroyed between 2015 2017 (Welcome, 2011).

Drug quality is primarily controlled by the National Agency for Food and Drug Administration and Control. The agency has established a Mobile Authentication Service. A team of girls from the Regina Pacis Secondary School in Onitsha devised a better technological solution, an app called the FD Detector which uses barcode technology to verify drug authenticity and expiration dates. This won them a place in the Technovation Challenge 2018. 
Several major regulatory failures have produced international scandals:

- In 1993, adulterated paracetamol syrup entered into the healthcare system in Oyo and Benue State, the end result of was the death of 100 children. A year after the disaster, batches containing poisonous ethylene glycol, the major cause of the deaths, could still be purchased.

- In 1996, about 11 children died of contamination from an experimental trial of the drug trovafloxacin.

- In 2008-2009, at least 84 children died from a brand of contaminated teething medication.

Healthcare in Nigeria is influenced by different local and regional factors that impacts the quality or quantity present in one location. Due to the aforementioned, the healthcare system in Nigeria has shown spatial variation in terms of availability and quality of facilities in relation to need. However, this is largely as a result of the level of state and local government involvement and investment in health care programs and education. Also, the Nigerian Ministry of Health usually spend about $70 \%$ of its budget in urban areas where around $50 \%$ of the population resides. Migration of health care personnel to other countries is a tasking and relevant issue in the health care system of the country. From a supply push factor, a resulting rise in exodus of nurses may be due to dramatic factors that make the work unbearable and knowing and presenting changes to arrest the factors may stem a tide.

Because a large number of nurses and doctors migrating abroad benefited from government funds for education, it poses a challenge to the patriotic identity of citizens and also the rate of return of federal funding of health care education. The state of healthcare in Nigeria has been worsened by a physician shortage as a consequence of severe 'brain drain' and retaining health care professionals is an important objective. Many Nigerian doctors have emigrated to North America and Europe. In 2005, 2,392 Nigeria doctors were practicing in the US alone, in UK number was 1,529. Retaining these expensively trained professionals has been identified as an urgent goal. The brain drain cut across all healthcare Professionals, thousands of Nigerian Pharmacists and Nurses are practicing in the UK and USA as well and so on. Empirical evidences reveal negative impact of commercialization of public health service delivery on attainment of the MDGs in Nigeria.

As recent reports have shown, in addition to the many benefits there are also risks associated with the different types of Traditional Medicine/Complementary or Alternative Medicine (TM/CAM). Although consumers today have widespread access to various TM/CAM treatments and therapies, they often do not have enough information on what to check when using TM/CAM in order to avoid unnecessary harm. While traditional medicine has a lot to contribute to the health and economy, much harm has resulted from unregulated sale and misuse of traditional/alternative medicine and herbs in the country and has significantly delayed patients' seeking professional healthcare.

The quality of Nigerian healthcare institutions is generally considered rather poor. There might also be shortages of medical supplies, and the blood supply of the country is not considered safe. Hygiene conditions are problematic, especially outside the large cities (Welcome, 2011). A major problem afflicting the healthcare system in Nigeria is the so-called "brain drain" of doctors and medical staff. It is estimated that there are four doctors for every 10,000 inhabitants. Highly-trained experts often leave the country in order to pursue their profession in countries with better infrastructure or higher wages. 
However, Nigeria has one of the largest stocks of Human Resources for Health (HRH) in Africa but, like the other $57 \mathrm{HRH}$ crisis countries, has densities of nurses, midwives and doctors that are still too low to effectively deliver essential health services (1.95 per 1,000). In recent years migration to foreign countries has declined and the primary challenge for Nigeria is inadequate production and inequitable distribution of health workers. The health workforce is concentrated in urban tertiary health care services delivery in the southern part of the country, particularly in Lagos (Aregbeshola, 2019).

This inequity has been attributed to: Lack of public and private sector coordination, favoring indigenous hires, commercial pressures in the private sector that lead to poor quality work, work environments that contribute to low motivation, less-than-optimal productivity, high attrition especially from rural areas; and lack of planning based on staffing projection needs resulting in an overproduction of some categories of health workers and a lack of others (Aregbeshola, 2019).

These challenges are further compounded by the fact that the federal government accepts and regulates 3 systems of health care delivery: orthodox, alternative, and traditional. The absence of a common $\mathrm{HRH}$ and data collection system leads to a lack of coordination in collecting $\mathrm{HRH}$ information, which means various stakeholders get fragmented information. Current strategies to manage $\mathrm{HRH}$ maldistribution and attrition include: use of telemedicine, financing/aid arrangements with other countries where significant populations of Nigerian health workers live plus efforts to enable easier transition to Nigeria; and actively managing brain drain.

One successful approach is the Midwifery Service Scheme, 2009. It mobilizes unemployed and retired but able midwives and newly qualified graduates from Nigerian Schools of Midwifery to rural communities for 1 year of community service.

\subsection{Key objectives of the current HRH policy include:}

- Providing a framework for objective analysis;

- Implementing and monitoring measures;

- Aligning health worker supply with health sector needs;

- Applying best practices to HRH management and development to promote equitable distribution and retention of the quality and quantity of $\mathrm{HRH}$ to ensure universal access to quality health services;

- Institutionalizing performance incentives and management systems that recognize hard work and service in deprived and unpopular locations;

- Fostering collaboration among public sector, non-government providers of health services and other HRH stakeholders; and strengthening the institutional framework for $\mathrm{HRH}$ management practices. Some states, such as Jigawa, already have begun adopting these national policies based on their needs. National HRH policies are expected to be adopted universally in Nigeria by the end of 2015 Aregbeshola (2019).

\subsection{Internet Of Things (loT)}

The availability of Internet of Things (IoT) is very vital in promoting rapid socio-economic and development of a nation. The Internet of Things refers to a type of network to connect anything with the Internet based on stipulated protocols through information sensing equipment to conduct information exchange and communications in order to achieve smart recognitions, positioning, tracing, monitoring, and administration. 
The IOT concept was coined by a member of the Radio Frequency Identification (RFID) development community in 1999, and it has recently become more relevant to the practical world largely because of the growth of mobile devices, embedded and ubiquitous communication, cloud computing and data analytics. Imagine a world where billions of objects can sense, communicate and share information, all interconnected over public or private Internet Protocol (IP) networks. These interconnected objects have data regularly collected, analyzed and used to initiate action, providing a wealth of intelligence for planning, management and decision making. This is the world of the Internet of Things.

Internet of Things (loT) is a network of physical objects. The Internet is not only a network of computers, but it has evolved into a network of devices of all type and sizes, vehicles, smart phones, home appliances, toys, cameras, medical instruments and industrial systems, animals, people, buildings, all connected, all communicating and sharing information based on stipulated protocols in order to achieve smart reorganizations, positioning, tracing, safety and control, even personal real time online monitoring, online upgrade, process control and administration (Ovidiu et al 2014).

We define loT into three categories as below:

Internet of Things is an Internet of three things: People to people, People to machine/Things and Things/machine to Things/machine, interacting through the Internet.

\subsection{Enabling Technologies For loT}

Internet of Things (IOT) is a global infrastructure for the information society, enabling advanced services by interconnecting (physical and virtual) things based on existing and evolving interoperable information and communication technologies. With the Internet of Things the communication is extended via Internet to all the things that surround us. The Internet of Things is much more than machine to machine communication, wireless sensor networks, sensor networks, 2G/3G/4G,GSM,GPRS,RFID, WI-FI, GPS, microcontroller, microprocessor etc. These are considered as being the enabling technologies that make "Internet of Things" applications possible.

Enabling technologies for the Internet of Things can be grouped into three categories:

Technologies that enable "things" to acquire contextual information, technologies that enable "things" to process contextual information, and technologies to improve security and privacy.

The first two categories can be jointly understood as functional building blocks required building "intelligence" into "things", which are indeed the features that differentiate the loT from the usual Internet. The third category is not a functional but rather a de facto requirement, without which the penetration of the loT would be severely reduced. (Ovidiu et al 2014).

The Internet of Things is not a single technology, but it is a mixture of different hardware and software technology. The Internet of Things provides solutions based on the integration of information technology, which refers to hardware and software used to store, retrieve, and process data and communications technology which includes electronic systems used for communication between individuals or groups. 
The fundamental characteristics of the loT are as follows:

Interconnectivity: With regard to the loT, anything can be interconnected with the global information and communication infrastructure.

Things-related services: The IoT is capable of providing thing-related services within the constraints of things, such as privacy protection and semantic consistency between physical things and their associated virtual things. In order to provide thing-related services within the constraints of things, both the technologies in physical world and information world will change.

Heterogeneity: The devices in the loT are heterogeneous as based on different hardware platforms and networks. They can interact with other devices or service platforms through different networks.

Dynamic changes: The state of devices change dynamically, e.g., sleeping and waking up, connected and/or disconnected as well as the context of devices including location and speed. Moreover, the number of devices can change dynamically.

Enormous scale: The number of devices that need to be managed and that communicate with each other will be at least an order of magnitude larger than the devices connected to the current Internet.

Even more critical will be the management of the data generated and their interpretation for application purposes. This relates to semantics of data, as well as efficient data handling.

Safety: As we gain benefits from the loT, we must not forget about safety. As both the creators and recipients of the loT, we must design for safety. This includes the safety of our personal data and the safety of our physical well-being. Securing the endpoints, the networks, and the data moving across all of it means creating a security paradigm that will scale.

Connectivity: Connectivity enables network accessibility and compatibility. Accessibility is getting on a network while compatibility provides the common ability to consume and produce data. IoT architecture consists of different layers of technologies supporting loT. It serves to illustrate how various technologies relate to each other and to communicate the scalability, modularity and configuration of loT deployments in different scenarios. The functionality of each layer is described below (Ovidiu et al 2013).

\subsubsection{Smart device/sensor layer:}

The lowest layer is made up of smart objects integrated with sensors. The sensors enable the interconnection of the physical and digital worlds allowing real-time information to be collected and processed. There are various types of sensors for different purposes. The sensors have the capacity to take measurements such as temperature, air quality, speed, humidity, pressure, flow, movement and electricity etc. In some cases, they may also have a degree of memory, enabling them to record a certain number of measurements. A sensor can measure the physical property and convert it into signal that can be understood by an instrument. Sensors are grouped according to their unique purpose such as environmental sensors, body sensors, home appliance sensors and vehicle telematics sensors, etc. 
Most sensors require connectivity to the sensor gateways. This can be in the form of a Local Area Network (LAN) such as Ethernet and Wi-Fi connections or Personal Area Network (PAN) such as ZigBee, Bluetooth and Ultra-Wideband (UWB). For sensors that do not require connectivity to sensor aggregators, their connectivity to backend servers/applications can be provided using Wide Area Network (WAN) such as GSM, GPRS and LTE. Sensors that use low power and low data rate connectivity, they typically form networks commonly known as wireless sensor networks (WSNs). WSNs are gaining popularity as they can accommodate far more sensor nodes while retaining adequate battery life and covering large areas.

\subsubsection{Gateways and Networks:}

Massive volume of data will be produced by these tiny sensors and this requires a robust and high performance wired or wireless network infrastructure as a transport medium. Current networks, often tied with very different protocols, have been used to support machine-tomachine (M2M) networks and their applications. With demand needed to serve a wider range of IoT services and applications such as high speed transactional services, context-aware applications etc., multiple networks with various technologies and access protocols are needed to work with each other in a heterogeneous configuration. These networks can be in the form of a private, public or hybrid models and are built to support the communication requirements for latency, bandwidth or security. Various gateways (microcontroller, microprocessor.) and gateway networks (WI-FI, GSM, GPRS...) VoiP Trunk Gateway, Network Gateway, Internet-To-Orbit Gateway, Media Gateway, Payment Gateway, Default Gateway, IoT Gateway, Cloud Storage Gateway.

\subsubsection{Management Service Layer}

The management service renders the processing of information possible through analytics, security controls, process modeling and management of devices. One of the important features of the management service layer is the business and process rule engines. loT brings connection and interaction of objects and systems together providing information in the form of events or contextual data such as temperature of goods, current location and traffic data. Some of these events require filtering or routing to post-processing systems such as capturing of periodic sensory data, while others require response to the immediate situations such as reacting to emergencies on patient's health conditions. The rule engines support the formulation of decision logics and trigger interactive and automated processes to enable a more responsive loT system. Data management is the ability to manage data information flow. With data management in the management service layer, information can be accessed, integrated and controlled. Higher layer applications can be shielded from the need to process unnecessary data and reduce the risk of privacy disclosure of the data source.

\subsubsection{Application Layer}

The loT application covers "smart" environments/spaces in domains such as: Transportation, Building, City, Lifestyle, Retail, Agriculture, Factory, Supply chain, Emergency, Healthcare, User interaction, Culture and tourism, Environment and Energy.

\section{ANALYSIS OF THE PROPOSED SYSTEM}

The proposed system is a web based application which by extension it is expected to address issues related to the limitation of the existing system such as speed of operation, poor security, issues of documentation and recovery of information. 


\subsection{System Design Approach}

The proposed system is an automated system used for documentation of medical records, scheduling of appointments with doctors on different specialty. It is a computer software that works using database system linked to the application and so enhancing the documentation, storage and retrieval of medical records.

To ensure efficiency in health care system one has to create a prototype to gather requirements and such that the user can identify the faults or errors in the prototype. The Researcher adopted the rapid prototype for my design and the methodology because rapid prototyping involves the software developer to gather the necessary information in the requirement process to build a reduce version of the system in question so as to enable him gather real and exact need of patients. Rapid prototyping is a method used to overcome the issues related to understanding and capturing the user's requirements.

Reasons for choosing the model;

1. It enables the developer to benefit from the experience gained from building the prototype and apply this experience towards building a better system.

2. It gathers client's feedback early in the process to avoid cost in redesign.

3. It demonstrate technical feasibility when the technical risk is high and provide early functionality.

4. It can also be used to have better understanding and extract user requirements and its documentation focuses on the end product not the evolution of the product

\subsection{Choice Of Tools}

The choice of programming tools used in developing the software is;

i. $\quad$ PHP and MySql (backend)

ii. HTML5 CSS3 and JQUERY (frontend)

\subsubsection{PHP and MySql:}

PHP is the most popular scripting language for web development. It is free, open source and server-side (the code is executed on the server)scripting language. MySQL is a Relational Database Management System (RDBMS) that uses Structured Query Language (SQL). It is also free and open source. The combination of PHP and MySQL gives unmet options to create just about any kind of website - from small contact form to large corporate portal.

PHP was at first created as a simple scripting platform called "Personal Home Page". Nowadays PHP is an alternative of the Microsoft Active Server Pages (ASP) technology. PHP is an open source server-side language which is used for creating dynamic web pages. It can be embedded into HTML. PHP is usually used in conjunction with a MySQL database on Linux/UNIX web servers. It is probably the most popular scripting language. PHP is a widely-used generalpurpose scripting language and interpreter that is freely available.

MySQL is a freely available open source Relational Database Management System (RDBMS) that uses Structured Query Language (SQL).

SQL is the most popular language for adding, accessing and managing content in a database. It is noted for its quick processing, proven reliability, ease and flexibility of use. MySQL is an essential part of almost every open source PHP application. Good examples for PHP \& MySQLbased scripts are WordPress, Joomla, Magento and Drupal.(11). 


\subsubsection{HTML5 CSS3 and JQUERY}

HTML5 and CSS3 are the latest versions of HTML and CSS, and are now part of everyday web design and development. They bring tons of rich features which allows you to make beautiful looking web pages much more easier than before.

JQuery is a Javascript library (canned javascripts) that make it really easy to create all kinds of cool web widgets. Like HTML5 and CSS3, JQuery is used in everyday web design and retrieval of medical records.

\subsection{Functional and Non-Functional Requirements}

The proposed system is designed to help manage the healthcare system, tackle issues of queues and properly enhance patient and doctor's private communication, schedule of appointments, and also prepare the database for monitoring doctor's routine and how well they respond to these patients.

In addition, the system will also be able to achieve the following;

- $\quad$ Determine how doctors respond to patients.

- $\quad$ Keep records of the patients who visited the doctor in the database.

- Handle challenges that pose queues at the hospital.

- Know which doctor attended to a particular patient.

Non functional requirements for the system are:

- User friendly interface, so users can navigate the system effectively.

- Tracking the activity mechanism so the management can evaluate the usage of the system.

- $\quad$ Ensure faster response as it will prompt efficiency in the process.

- It will enable management to know how well a doctor responded to a patient.

\subsection{System Input}

The system input in this case refers to the all the fields required to process the medical records Of patients, scheduling of appointment with doctors, and the doctors responds to this appointments.

\section{Input For Patients}

The patient gives clear and detailed information about his or herself such as the name, address, city, gender, phone number, email, state and local government area.

\section{Input For Doctors}

Here the doctors gives their information as well, such as name, email and specialty. 


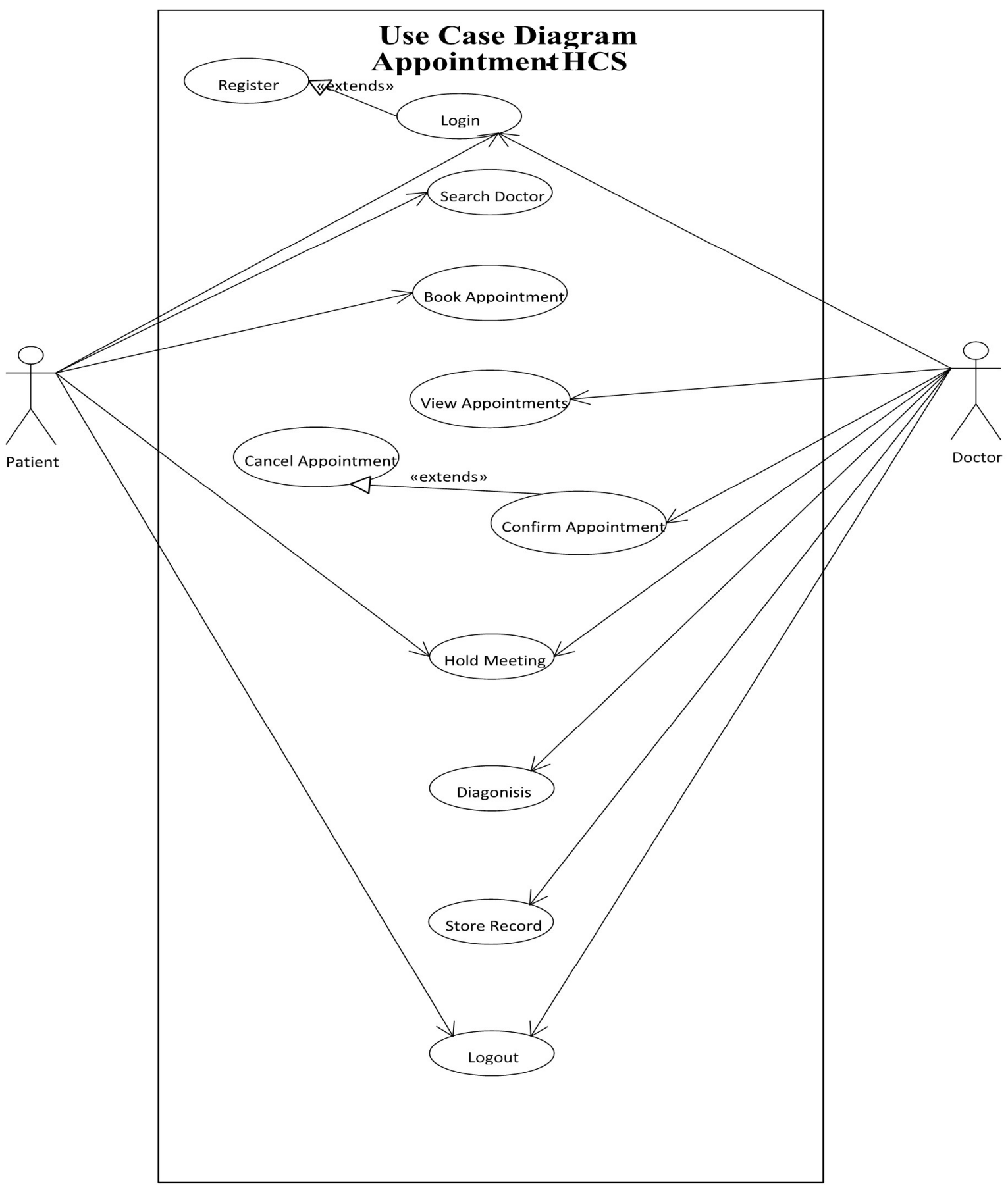

Fig. 2 Use Case Diagram for Scheduling Appointment.

This shows how patients schedule appointment based on the specialist they are seeing, the specialist will view the appointment and confirm the date and time before meeting can hold. 


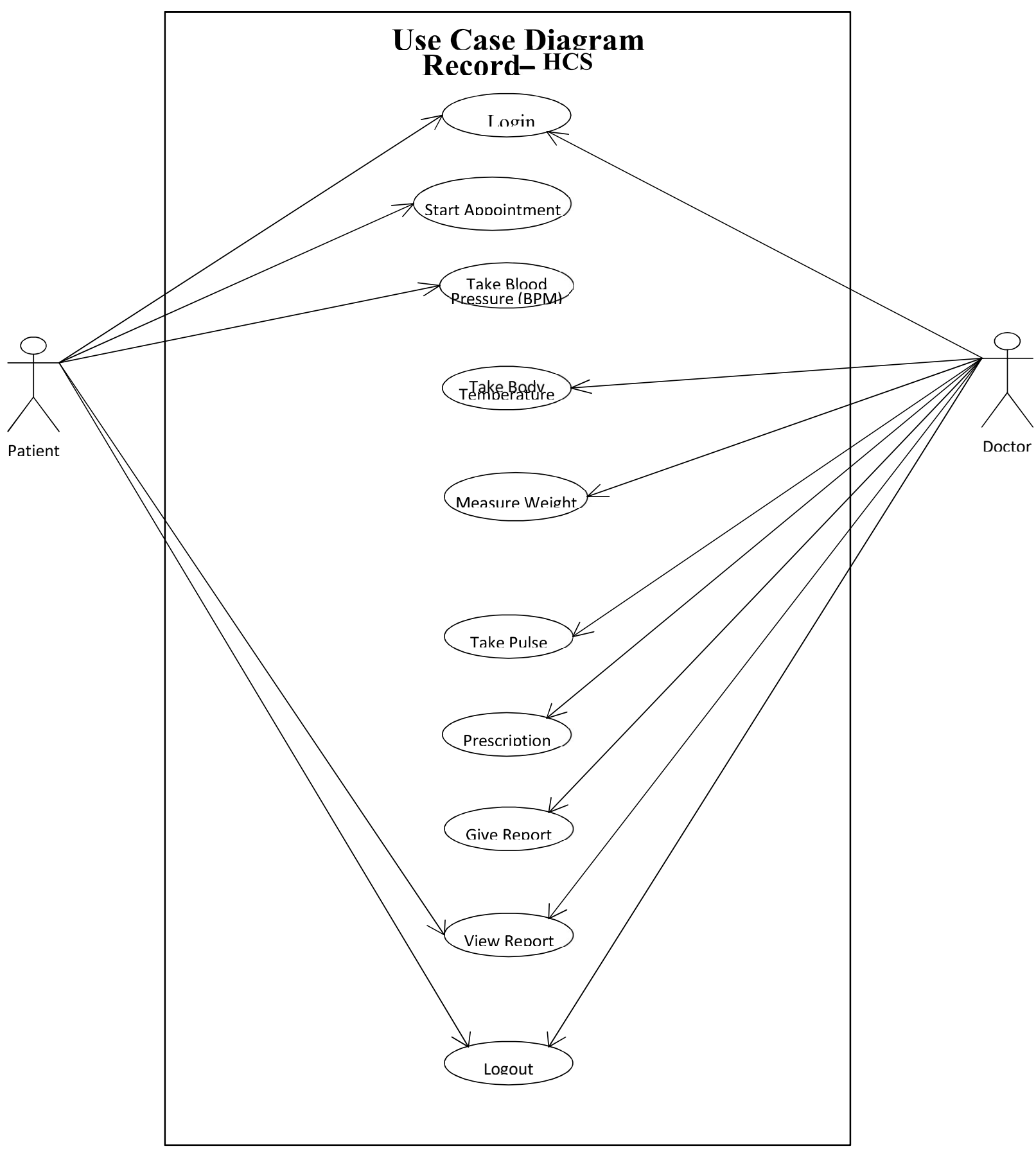

Fig. 3 Use Case Diagram for Records

Shows doctor's action and report after confirmation to patient's appointment and examining the patient. 
Class Diagram

Health Care System (HCS)

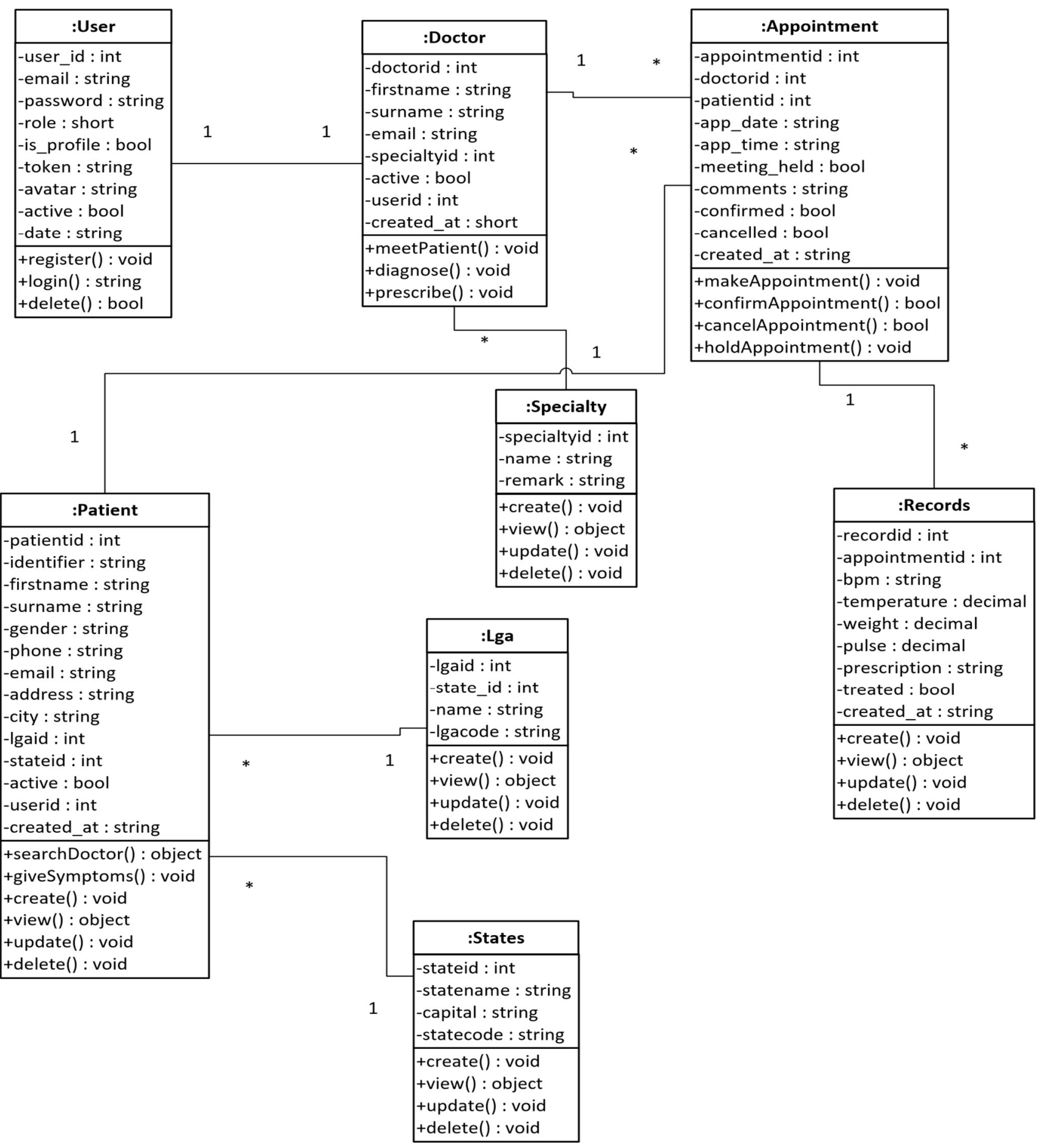

Fig. 4 Class Diagram for Health Care System 


\section{SYSTEM STRUCTURAL CHART}

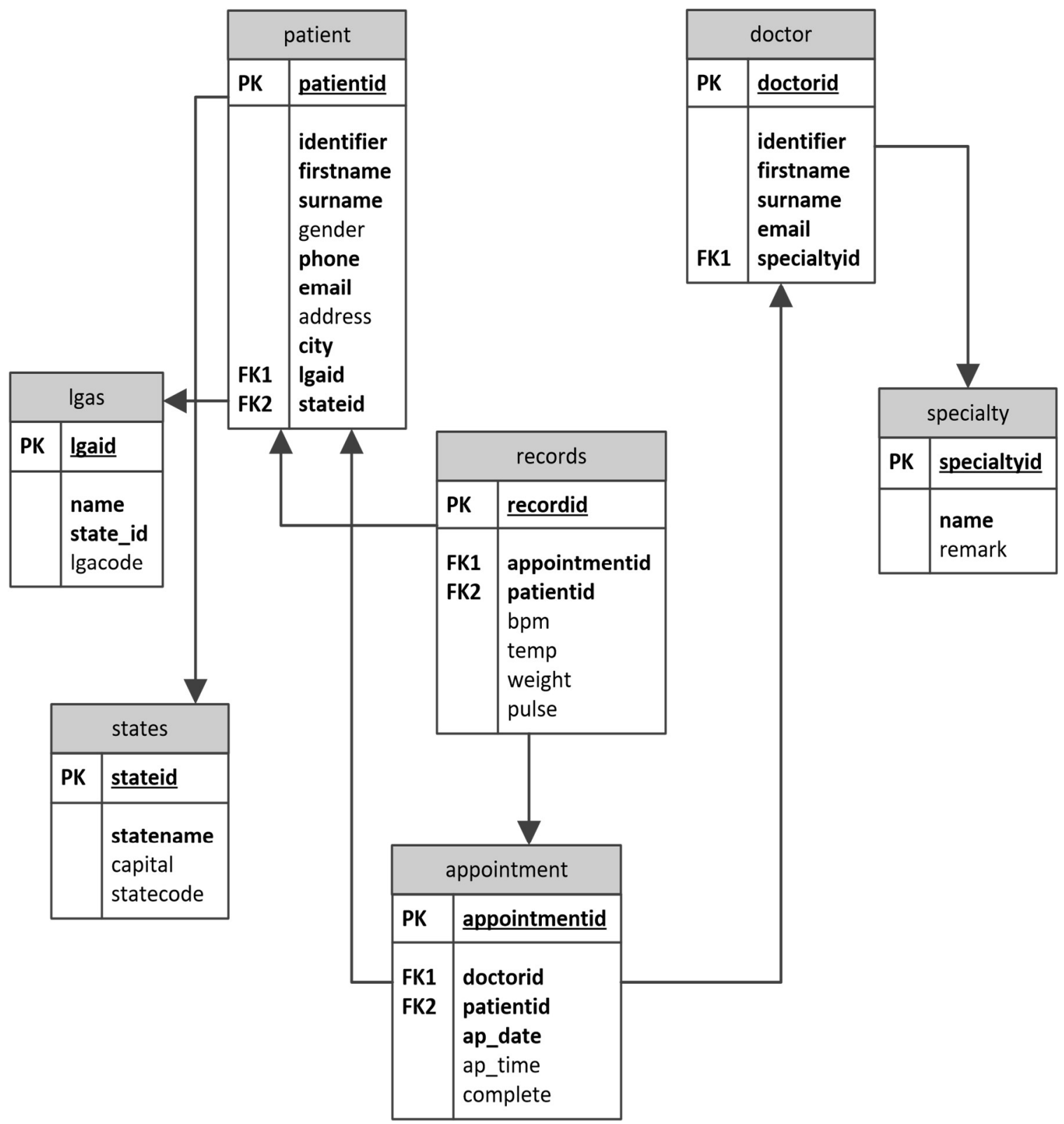

Fig. 5 System Structural Chart

Shows Entity-Relationship in the Health Care System (HCS) 


\section{REFERENCES}

1. Aregbeshola, Bolaji (2019) Health care in Nigeria: Challenges and recommendations https://socialprotection.org/discover/blog/health-care-nigeria-challenges-andrecommendations

2. Browne, S G (2017) A History of the Nigerian Health Services https://www.ncbi.nlm.nih.gov/pmc/articles/PMC1081514/?page=1 and page 2

3. DHS Documentation (2016) Strategic Principles For Securing The Internet Of Things (IOT) Version 1.0 U.S. Department of Homeland Security (DHS)

4. Enisa Documentation (2017) Baseline Security Recommendation of IoT; in context of critical information Infrastructures www.enisa.europa.eu Europea Union Agency For Network And Information Security (ENISA)

5. Goldman Sachs, David H Roman, Kyle D, Conlee, (2015) Equity Research, Internet of Things, Vol. 5 The Digital Revolution comes to US Healthcare

6. Martín Serrano, Payam Barnaghi, Francois Carrez Philippe Cousin, Ovidiu Vermesan, Peter Friess, (2015) Internet of Things Semantic Interoperability: Research Challenges, Best Practices, Recommendations and Next Steps, European research cluster on the internet of things, IERC.

7. Karen, Rose Scott Eldridge, Lyman Chapin, (2015) "The Internet of Things: An Overview Understanding the Issues and Challenges of a More Connected World", The Internet Society (ISOC).

8. Ohuabunwa, M. (2002). Health care delivery in Nigeria. Past Present and the future. Nigerian Journal of Pharmacy. 31:15-17.

9. Ovidiu Vermesan SINTEF, Norway, Peter FriessEU, Belgium,(2013) Internet of Things: Converging Technologies for Smart Environments and Integrated Ecosystems, river publishers' series in communications.

10. Ovidiu Vermesan SINTEF, Norway, Peter FriessEU, Belgium (2014), Internet of ThingsFrom Research and Innovation to Market Deployment, river publishers' series in communications.

11. Rowe, Michael (2008). Information and Communication Technology in Health, A review literature. University of the Western Cape. https://www.researchgate.net/publication/220009616_Information_and_communica tion_technology_in_health_A_review_of_the_literature

12. Welcome, M.O (2011). The Nigerian health care system: need for integrating adequate medical intelligence and surveillance systems, J Pharm Bioallied Sci; 3(4):470-478. 